\title{
Encore
}

IgM rheumatoid factor in juvenile rheumatoid arthritis The prevalence of IgM rheumatoid factor in children with arthritis has been hard to determine for sure. A recent study in 68 children with active disease using an enzyme linked immunosorbent assay (ELISA) technique has shown that IgM rheumatoid factor is present in two thirds of those with active polyarticular disease, in about a quarter of those with systemic disease, and in only $6 \%$ of those with the pauciarticular form. It is usually associated with the presence of severe disease.

f Rheumatol 1990; 17: 936-40.

Survival of children with systemic lupus erythematosus (SLE)

It is particularly distressing to see SLE in children. How do they fare? Apparently about $85 \%$ survive for five years from the onset of the disease but at a significant cost from complications of the treatment and damage to organs such as the brain, lungs, and kidneys.

f Rheumatol 1990; 17: 941-5.

\section{The right to die}

Is it ethically right to switch off the ventilator when the patient is reduced to the status of a vegetable to the evident distress of the relatives? The United States Supreme Court in its decision over the Nancy Cruzan case affirmed the patient's right to refuse life sustaining treatment. Where no such right was claimed by the patient before illness removed the ability to make decisions, however, the question of whether to continue treatment or not remains one for the doctors' ethics and the relatives' informed wishes. Thirty five doctors gave their signatures to just such a statement. This is a matter for the conscience of all doctors. $N$ Engl f Med 1990; 323: 686-7.

Antiperinuclear factor antigen and rheumatoid arthritis (RA)

The presence of the antiperinuclear factor antigen has been thought to be fairly specific for RA, but recent studies have thrown some doubt on this. It has also been discovered in seronegative RA, Sjögren's syndrome, other rheumatic diseases, and even in healthy controls. A recent study suggests that the antiperinuclear factor may indeed recognise a common autoantigen found generally in connective tissue disease.

Arthritis Rheum 1990; 33: 960-9.

Ankylosing spondylitis (AS) and the ability to work So often patients with AS ask what they can and cannot safely do, and their employers certainly have distinct prejudices about it. A recent study from France suggests that they do badly if there is much peripheral joint disease or if they have to carry heavy loads. Working in the cold or having to stand for long periods was inadvisable too, but sedentary work and appropriate rehabilitation regimens seemed to be protective.

Arthritis Rheum 1990; 33: 1001-6.

Are the poor unhealthy?

Yes, undoubtedly, the evidence quoted by a leader in the $B M \mathcal{F}$ indicates. One way that their health can be changed for the better is to improve their economic state. It is a sad reflection on modern British society that the disparity in income and health status seems to be increasing between the comfortably off and the poor. This is less evident in Scandinavia. The clear implication is to bring the income of the poor nearer to that of the wealthy, not the other way round. A relatively prosperous Britain does not seem to be doing it.

$B M \mathcal{F}$ 1990; 301: 349-50.

\section{Systemic amyloidosis and its evaluation}

The presence and extent of systemic amyloidosis can be difficult to evaluate and diagnose and it usually means the patient undergoing a biopsy. Scintigraphy using the injection of ${ }^{123} \mathrm{I}$ labelled serum amyloid $P$ component has now been shown to be an effective method for finding systemic amyloid, measuring its extent, and seeing what happens to it.

N Engl f Med 1990; 323: 508-13.

\section{Gout and hyperuricaemia in the young}

Gout may be seen in young and relatively young men and women. They seem to have a below normal renal uric acid clearance, which may be due to a specific renal tubular defect. Some of them have short, stubby fingers and are short in height with an abnormal facial appearance. This seems to indicate a distinct syndrome affecting the skeletal appearance and renal function. Young people with gout should have their urate handling studied the authors conclude.

QF Med 1990; 75: 441-50.

\section{Waiting time for outpatients}

Patients often have to wait for an unacceptably long time before seeing the doctor in outpatient clinics, though this is frequently unavoidable for a mixture of reasons. I thought this was a particularly British disease, and politicians certainly seem to think so. To my surprise an article from the United States (where medicine is organised very differently) suggests that waiting is not unknown there either. One thing is clear, there are many things we can and should be doing to shorten waiting times.

$N$ Engl f Med 1990; 323: 604-8.

\section{Salivary epidermal growth factor and RA}

A recent study suggests that epidermal growth factor production is reduced in RA, and this factor is important in the defence and repair of the gastric mucosa. We give our patients with RA non-steroidal anti-inflammatory drugs, which by reducing gastric mucosa prostaglandins remove yet another protective mechanism against gastric ulceration. Perhaps this goes some way towards explaining why patients with this form of arthritis so often get ulcers.

$B M \mathcal{F}$ 1990; 301: 422-3.

\section{Radiation exposure of patients}

About $90 \%$ of exposure to man-made ionising radiation comes from diagnostic radiology, so any way to reduce this is welcome. In the first place about one fifth of radiographs are unnecessary and we do not use alternative measures of imaging as much as we should. Yet another argument for better provision and use of ultrasound and, in particular, rare earth screens in diagnostic radiography departments. The use of magnetic resonance imaging much more widely would be even better and Britain lags behind in this respect. BMF 1990; 301: 451-2.

\section{Elbow arthroplasty in RA}

We are referring patients with $\mathrm{RA}$ and severely diseased elbows increasingly to our orthopaedic colleagues. But how good is the operation? Good it would seem at least in the short term. Flexion range increased, hand function improved, and there was much less pain. Two of 20 developed ulnar nerve paraesthesia and one an epicondylar fissure, however. Acta Orthop Scand 1990; 61: 344-7. 